\title{
Interaction of retention interval with CS-preexposure and extinction treatments: Symmetry with respect to primacy
}

\author{
DANIEL S. WHEELER, STEVEN C. STOUT, and RALPH R. MILLER \\ State University of New York, Binghamton, New York
}

\begin{abstract}
Imposition of a retention interval between cue-outcome pairings and testing can alleviate the retardation of conditioned responding induced by pretraining exposure to the cue (i.e., the CS-preexposure effect). However, recent studies have reported an enhanced effect of CS-preexposure treatment with longer retention intervals (De la Casa \& Lubow, 2000, 2002; Lubow \& De la Casa, 2002). In a series of conditioned barpress suppression studies with rats, we examined the effects of imposing a retention interval just prior to testing following either CS-preexposure (cue alone before cue-outcome pairings) or extinction (cue alone after cue-outcome pairings) treatments. Experiment 1 replicated in a different preparation recent reports of CS-preexposure treatment effects increasing with longer retention intervals. Experiment 2 showed that spontaneous recovery of stimulus control of behavior after extinction can be obtained with the same parameters as those used to observe the augmented effect of CS-preexposure treatment. In Experiment 3, both the augmented effect of CS-preexposure treatment and spontaneous recovery from extinction were found when we used, in place of a retention interval, an associative priming manipulation.
\end{abstract}

The CS-preexposure effect refers to the retardation of responding to a conditioned stimulus (CS) that is observed when the subject has been exposed to that CS alone before the CS is paired with the unconditioned stimulus (US). Numerous theories predict the effect of CS preexposure, and alternative hypothetical mechanisms include a learning deficit resulting from a decrease in associability or attention to the CS (e.g., Lubow, Schnur, \& Rifkin, 1976; Mackintosh, 1975; Pearce \& Hall, 1980), a response deficit due to a robust CS-context association (Grahame, Barnet, Gunther, \& Miller, 1994), and a response deficit arising from interfering associations to the CS (e.g., Bouton, 1993; Miller, Kasprow, \& Schachtman, 1986; Spear, 1978). Efforts to test these theories have yielded a large number of empirical findings concerning the nature of the CS-preexposure effect. The present series of experiments deals specifically with posttraining manipulations that may produce a recovery from the effect of CS-preexposure treatment, which suggests that CS preexposure does not lead to an irrevocable retardation of acquisition, but instead, a temporary retardation of responding to the target CS.

Experiments investigating posttraining manipulations and the effect of CS-preexposure treatment have shown that a retention interval interposed between conditioning and testing can result in a decrease in the CS-preexposure

Support for this research was provided by NIMH Grants 33881 and 644202. We thank Jim Esposito for his technical assistance, and Jeffrey Amundson, Oskar Pineño, Gonzalo Urcelay, and Kouji Urushihara for comments on an earlier version of the manuscript. Correspondence should be addressed to R. R. Miller, Department of Psychology, SUNY-Binghamton, Binghamton, NY 13902-6000 (e-mail: rmiller@binghamton.edu). effect - that is, a recovery of conditioned responding (e.g., Killcross, Kiernan, Dwyer, \& Westbrook, 1998). (This should not be confused with a similar effect that may be observed when a retention interval is imposed between CS preexposure and conditioning.) This recovery from the CS-preexposure effect has been likened to the spontaneous recovery effect seen when an extinguished CS is tested after a long retention interval (e.g., Pavlov, 1927). This similarity (among others) between the CSpreexposure effect and extinction has contributed to theories suggesting that the effect of CS-preexposure treatment, like that of extinction, is not a permanent impairment of responding, but a process involving interference between the expression of reinforced and nonreinforced memories of a stimulus (e.g., Bouton, 1993; Spear, 1978). (There are also attention-focused theories of both the CSpreexposure effect and extinction; these are addressed in the General Discussion.) Although not as widely reported as spontaneous recovery from extinction, recovery of conditioned responding following CS-preexposure and conditioning treatments has been obtained in a number of experiments (e.g., Aguado, Symonds, \& Hall, 1994; Killcross et al., 1998; Kraemer, Randall, \& Carbary, 1991; Kraemer \& Roberts, 1984; for conflicting results, see, e.g., De la Casa \& Lubow, 2000; Kraemer \& Ossenkopp, 1986).

The retrieval-deficit theory of the CS-preexposure effect (e.g., Spear, 1978) cannot fully explain the recovery of responding with the passage of time; the theory can only explain why a recovery is possible. Specifically, the theory requires a mechanism to account for shifts in interference between different memories. Toward providing a cognitive mechanism by which a recovery of re- 
sponding could occur, Bouton (1993) has suggested a retrieval-interference theory in which memory expression is mediated by the context of testing. According to this theory, subjects acquire two different types of CS memories when a stimulus is first exposed alone, and then paired with a US. These memories are contradictory and compete for expression when the stimulus is presented at test. Also, the expression of a given type of memory (e.g., reinforced or unreinforced) is facilitated by context cues. Furthermore, the expression of the different types of CS memories may be differentially dependent upon occasion setting by the context. Bouton has suggested that when a CS is presented after unreinforced followed by reinforced presentations, the subject's response to the CS is influenced by contextual cues, which can include features of the physical or temporal context that are associated with particular information pertaining to the CS. Therefore, the theory predicts that animals trained and soon afterward tested in a consistent physical context will show a recency effect; responding will be congruent with recently acquired memories, the retrieval of which is facilitated by the recent temporal context. However, when the CS is presented in a novel context (physical or temporal), there are fewer cues present to facilitate retrieval of either of the conflicting CS memories; therefore, responding will reflect the memories of the CS that are less reliant upon occasion setting by the context. In the aforementioned cases involving testing the effects of CS-preexposure (or extinction) treatment and conditioning after a shift in the temporal context (i.e., after a long retention interval; e.g., Aguado et al., 1994; Killcross et al., 1998; Kraemer et al., 1991; Kraemer \& Roberts, 1984), responding to the CS is augmented, reflecting enhanced expression of the memory of the reinforced trials. These results suggest that the reinforced memories of the CS are more durable or less context specific than the nonreinforced memories. These memories of reinforcement are presumably more important than memories of nonreinforcement because they involve inherently rewarding or aversive stimuli in most classical conditioning preparations.

In contrast to the findings indicating a recovery from the CS-preexposure effect with the passage of time, De la Casa and Lubow (2000, 2002; Lubow \& De la Casa, 2002) have observed an opposite effect. Using a conditioned taste aversion (CTA) preparation, they have repeatedly shown that a long retention interval interposed between training and testing can augment a CS-preexposure effect, producing a further decrement in conditioned responding. An augmented CS-preexposure effect is not compatible with the previously mentioned assumption that memories of reinforcement are more readily expressed than memories of nonreinforcement, but instead supports the assumption that first-learned memories of the CS are more readily expressed or less context specific than subsequent contradictory memories in a novel temporal context, regardless of reinforcement (Konorski \& Szwejkowska, 1952; also proposed in Bouton, 1993).
This principle of increasing primacy with delayed testing also accurately predicts spontaneous recovery after extinction: that the first-learned experiences (the reinforced trials in an extinction design) will be better expressed as the retention interval is increased.

Since first observing the augmented CS-preexposure effect, De la Casa and Lubow have completed considerable research examining the properties of the phenomenon. They have studied the importance of the US intensity (De la Casa \& Lubow, 2002), the time course over which the effect takes place (De la Casa \& Lubow, 2002), the importance of the retention interval context (De la Casa \& Lubow, 2000), and the parallelism with spontaneous recovery after extinction (Lubow \& De la Casa, 2002). In all, the effect is clearly replicable within their CTA preparation using their parameters. However, there are still a number of conflicting reports that suggest an opposite or null effect (e.g., Aguado et al., 1994; Kraemer \& Ossenkopp, 1986). Toward reconciling the conflicting reports, De la Casa and Lubow (2000) provided evidence suggesting that the physical context of the retention interval plays a critical role in the occurrence of the augmented CS-preexposure effect. In most CTA experiments, training and testing takes place in the home cages. Generally, in CTA studies, including those in which spontaneous recovery from the CS-preexposure effect has been observed in adult rats (e.g., Aguado et al., 1994), any retention interval also takes place in the home cage (i.e., the same context as training). De la Casa and Lubow found that, in their preparation, the augmented CS-preexposure effect occurs only when the retention interval is experienced outside of the experimental apparatus. According to some theories of learning (e.g., Dickinson \& Burke, 1996; Miller \& Matzel, 1988), and supported by empirical results (e.g., Escobar, Arcediano, \& Miller, 2002), extinguishing the training context should cause a recovery in responding to a target stimulus that was preexposed prior to conditioning (the opposite of an augmented CS-preexposure effect). Escobar et al.'s observation supports De la Casa and Lubow's explanation of the discrepancy between their result and the reports of recovery from the CS-preexposure effect (e.g., Aguado et al., 1994; Kraemer \& Roberts, 1984).

Some experiments, however, indicate that other factors must play a role in producing an augmented CSpreexposure effect. In reports by Killcross et al. (1998) and Kraemer et al. (1991), the context of the retention interval was different from the context of training, yet recovery from the CS-preexposure effect was still observed. Clearly, at least in the case of fear conditioning, the augmented CS-preexposure effect depends on more than simply the prevention of extinction of the experimental context. The fact that, to the best of our knowledge, an augmented CS-preexposure effect has not been observed in a Pavlovian conditioning preparation other than CTA leads to questions about the generality of the effect. The following set of experiments was conducted to determine whether an augmented CS-preexposure ef- 
fect can extend outside of the CTA preparation, and, if so, to further characterize the nature of the phenomenon.

\section{EXPERIMENT 1 Augmentation of the CS-Preexposure Effect}

The aim of Experiment 1 was to replicate the augmented CS-preexposure effect in a conditioned barpress suppression preparation. Because the primary goal of Experiment 1 was replication, the experiment was conducted using parameters that were expected to maximize the probability of obtaining the effect. This involved embedding the CS-preexposure procedure in a sensory preconditioning preparation during training. In this sensory preconditioning preparation, a CS (X) was trained with an innocuous outcome (i.e., a surrogate US, hereafter identified as $\mathrm{S}$ ). After $\mathrm{X} \rightarrow \mathrm{S}$ training, the $\mathrm{S}$ stimulus was reinforced with a US (footshock), which potentiated responding to the target $\mathrm{CS}, \mathrm{X}$, through a mediated associative link $(\mathrm{X} \rightarrow \mathrm{S} \rightarrow$ footshock $)$.

The rationale for our embedding this CS-preexposure experiment within a sensory preconditioning procedure was based on both theory and empirical results. As detailed in the introduction, theories that stress the durability of reinforced memories rely upon the notion that memories of reinforcement are more significant to an animal than memories of nonreinforcement, because memories of reinforcement often involve a highly appetitive or aversive event (e.g., Bouton, 1993; Kraemer \& Roberts, 1984). Logically, training a CS with an innocuous outcome (S) weakens the distinction in significance between the memories of the cue followed by the outcome and of the memories of the cue not followed by the outcome. Because conditioned responding is never evoked during $\mathrm{X} \rightarrow \mathrm{S}$ training, it is less likely that experiencing $\mathrm{X}$ followed by the surrogate outcome $(X \rightarrow S)$ would be much more significant than exposure to $\mathrm{X}$ without the surrogate outcome $(\mathrm{X}-)$. In support of this view, a study conducted in our laboratory with human subjects, which did not use biologically significant outcomes, revealed a shift from a recency effect to a primacy effect with increasing retention intervals after both CS-preexposure followed by conditioning and conditioning followed by extinction (i.e., spontaneous recovery; Stout, Amundson, \& Miller, 2004). Therefore, a sensory preconditioning preparation may be well suited for studying changes in responding that occur across retention intervals in that there are few differences in the importance of the conditioning trials (i.e., $\mathrm{X} \rightarrow \mathrm{S}$ ) and nonconditioning trials (i.e., $X-)$. Additionally, sensory preconditioning, with its lack of a primary reinforcer during target training, more closely approximates many human learning situations, which rely on higher order conditioning. That is, human learning often relies upon learning about inherently neutral outcomes (e.g., currency) that are only associated with inherently rewarding or aversive outcomes.

The design of Experiment 1 was also nontraditional in that the subjects in the CS-preexposure (PE) condition received an equal number of nonreinforced and reinforced presentations of the CS X. Studies of the CS-preexposure effect using a conditioned suppression preparation ordinarily use a much larger number of nonreinforced trials than reinforced trials (for a review, see Lubow, 1989), but we sought to produce a small CS-preexposure effect to allow for additional retardation of conditioned responding after a long retention interval. The subjects in the control (Ctrl) condition received preexposure to an irrelevant stimulus (Y), followed by pairings of CS X with S. After training with $\mathrm{X}$, the $\mathrm{S}$ outcome stimulus was paired with the footshock US. The test of X took place either 3 or 27 days following first-order conditioning of the surrogate $(\mathrm{S} \rightarrow \mathrm{US})$, for the subjects in the short and long conditions, respectively. It was expected that responding in the CSpreexposure condition would be sensitive to the change in retention interval, while responding would remain stable in the control condition.

\section{Method}

\section{Subjects}

The subjects were 24 male and 24 female experimentally naive Sprague-Dawley descended rats obtained from our own breeding colony. Body-weight ranges were 306-419 g for males and 196-290 g for females. The animals were individually housed in standard hanging stainless-steel wire-mesh cages in a vivarium maintained on a 16:8-h light:dark cycle. Experimental manipulations occurred near the middle portion of the light phase. The animals were allowed free access to Purina Lab Chow, whereas water availability was limited to $20 \mathrm{~min}$ per day following a progressive deprivation schedule initiated 1 week prior to the start of the study. From the time of weaning until the initiation of the study and during the long retention interval, all animals were handled for $30 \mathrm{sec}$, three times per week. Subjects were randomly assigned to one of four groups ( $n=12$ for each group), counterbalanced within groups for sex. The groups were designated preexposure-short retention interval (PEShort), preexposure-long retention interval (PE-Long), control-short retention interval (Ctrl-Short), and control-long retention interval (Ctrl-Long).

\section{Apparatus}

The apparatus consisted of 12 operant chambers each measuring $30.5 \times 27.5 \times 27.3 \mathrm{~cm}(1 \times \mathrm{w} \times \mathrm{h})$. All chambers had clear Plexiglas ceilings and side walls, and metal front and back walls. On one metal wall of each chamber, there was an operant lever. Adjacent to this lever was a niche $(4.5 \times 4.0 \times 4.5 \mathrm{~cm})$ centered $3.3 \mathrm{~cm}$ above the floor. A solenoid could deliver $0.04 \mathrm{ml}$ of water into a cup at the bottom of the niche. Chamber floors were 4-mm stainlesssteel grids spaced $1.7 \mathrm{~cm}$ apart center-to-center, connected with NE-2 neon bulbs, which allowed constant-current footshock to be delivered by means of a high-voltage AC circuit in series with a $1.0-\mathrm{M} \Omega$ resistor. All chambers were housed in individual soundand light-attenuating environmental chests. Three $45-\Omega$ speakers mounted on three different interior walls of each environmental chest could deliver a complex tone (consisting of $3000-\mathrm{Hz}$ and $3200-\mathrm{Hz}$ pure tones), a 6-per-second click train, and a white noise, all at $8 \mathrm{~dB}$ (C-scale) above the ambient background sound of $78 \mathrm{~dB}$, which was produced primarily by a ventilation fan. An overhead flashing (0.25:0.25-sec on:off) light stimulus could be provided by a $60-\mathrm{W}$ incandescent bulb. Each chamber was illuminated by a dim (No. 1820) houselight. Chamber assignments were counterbalanced within groups.

\section{Acclimation and Shaping}

Prior to conditioning, a 4-day acclimation and shaping regimen was provided to establish stable rates of barpressing for a water re- 
ward. Each of these acclimation days involved at least one 60-min session. To facilitate magazine training, the onset of the water delivery was accompanied by the onset of a 0.5 -sec white noise. On Day 1, a fixed-time 2-min schedule of noncontingent solenoid operation occurred concurrently with a continuous reinforcement schedule. Rats that made fewer than 10 barpresses on Day 1 were given another session later that day. On Day 2, the noncontingent water delivery was discontinued, and subjects were trained on the continuous reinforcement schedule alone. All subjects that recorded fewer than 10 responses on this day were later placed back in the chambers and hand-shaped through successive approximation. The sessions on Days 3 and 4 provided water on a variable interval 20 -sec (VI-20) schedule. On both of these days, all subjects responded with more than 50 leverpresses in a session, and no extra training sessions were given. The VI-20 schedule of reinforcement prevailed throughout the remainder of the experiment, including testing.

\section{Procedure}

Experiment 1 featured three phases of training, a retention interval, and a test (Table 1). Phase 1 involved unpaired stimulus preexposure, which was followed by paired sensory preconditioning in Phase 2. Surrogate inflation occurred in Phase 3, followed by testing after a long or short retention interval.

Phase 1: Preexposure. On Days 5 and 6, Phase 1 training occurred during daily 60 -min sessions. Each day included six stimulusonly presentations ( $\mathrm{X}$ in the $\mathrm{PE}$ groups and $\mathrm{Y}$ in the $\mathrm{Ctrl}$ groups) separated by $9( \pm 4.5)$-min intertrial intervals (ITIs). Cues X and $Y$ were clicks and complex tone, respectively, counterbalanced within groups. Cue presentations were $60 \mathrm{sec}$ in duration.

Phase 2: Sensory preconditioning. On Days 7 and 8, the sensory preconditioning treatment took place over daily 60 -min sessions. For all groups, each session included six $\mathrm{X} \rightarrow \mathrm{S}$ pairings separated by $9( \pm 4.5)$-min ITIs. The onset of the 5 -sec Stimulus $\mathrm{S}$ occurred at the termination of the $60-$ sec CS X.

Phase 3: First-order conditioning of the surrogate stimulus. On Day 9, all subjects experienced four $\mathrm{S} \rightarrow$ US pairings in a $60-\mathrm{min}$ session. These trials were separated by $15( \pm 7.5)$-min ITIs. Again, the onset of the US (0.5-sec, $0.5-\mathrm{mA}$ footshock) occurred at the termination of the 5 -sec surrogate stimulus.

Short retention interval reshaping and testing. On Days 10 and 11, the animals from the PE-Short and Ctrl-Short groups were given daily 60 -min sessions of uninterrupted barpressing to restabilize baseline performance. Weak responders (those subjects that made fewer than 50 responses in a session) were given an extra 60 min session in each day. The animals from the long retention interval groups were left in their home cages. On Day 12, subjects from the two short retention interval groups were tested for suppression to $\mathrm{X}$ in a 30-min test session. In each session, there were two unreinforced presentations of $\mathrm{X}$ ( $60 \mathrm{sec}$ in duration) separated by a 7 -min ITI (onset to onset). The first test occurred $4.5 \mathrm{~min}$ following entry into the operant chamber. The numbers of barpresses emitted during the $120 \mathrm{sec}$ immediately prior to the onset of each test trial CS and during the presence of each test trial CS were both recorded. A suppression ratio was calculated by dividing the total number of

Table 1

Design Summary of Experiment 1

\begin{tabular}{lcccrc}
\hline \multicolumn{1}{c}{ Group } & Phase 1 & Phase 2 & Phase 3 & $\begin{array}{r}\text { Retention } \\
\text { Interval }\end{array}$ & Test \\
\hline PE-Short & $12 \mathrm{X}-$ & $12 \mathrm{X} \rightarrow \mathrm{S}$ & $4 \mathrm{~S} \rightarrow \mathrm{US}$ & 3 days & $\mathrm{X}$ \\
PE-Long & $12 \mathrm{X}-$ & $12 \mathrm{X} \rightarrow \mathrm{S}$ & $4 \mathrm{~S} \rightarrow \mathrm{US}$ & 27 days & $\mathrm{X}$ \\
Ctrl-Short & $12 \mathrm{Y}-$ & $12 \mathrm{X} \rightarrow \mathrm{S}$ & $4 \mathrm{~S} \rightarrow \mathrm{US}$ & 3 days & $\mathrm{X}$ \\
Ctrl-Long & $12 \mathrm{Y}-$ & $12 \mathrm{X} \rightarrow \mathrm{S}$ & $4 \mathrm{~S} \rightarrow \mathrm{US}$ & 27 days & $\mathrm{X}$ \\
\hline
\end{tabular}

Note-PE, preexposure; Ctrl, control; X and Y, 60-sec tone or 60-sec clicker; S, 5-sec flashing light; US, footshock. Testing consisted of two nonreinforced presentations of the target cue. barpresses (BP) made during the two CS presentations by the sum of that number plus half the total number of barpresses made during the 120 -sec intervals that immediately preceded each 60 -sec CS (i.e., $\mathrm{BP}_{\mathrm{cs}} /\left[\mathrm{BP}_{\mathrm{cs}}+0.5 \mathrm{BP}\right.$ pre-cs $]$ ). We used 2-min baseline measures as opposed to 1-min measures on the assumption that a larger time sample would better assess average rates of responding.

Long retention interval reshaping and testing. On Days 34 and 35 , subjects in the long retention interval condition were given 2 days of reshaping. Once again, extra 60-min sessions were given within a day to facilitate reshaping in weak responders. On Day 36, Groups PE-Long and Ctrl-Long were tested for suppression to X. The testing procedure was identical to the one used for the short retention interval groups.

\section{Results and Discussion}

Before analyzing the test trial suppression ratios, we examined the pre-CS scores to determine whether there were any differences between the groups with respect to baseline barpressing (i.e., differential fear of the context). The pre-CS scores were the total number of barpresses made by a subject in both 2-min periods leading up to the two CS presentations on each test day. The results of a 2 (treatment: PE vs. Ctrl) $\times 2$ (retention interval: short vs. long) analysis of variance (ANOVA) showed no significant main effect or interaction $(p s>.25)$, indicating that any group differences in fear expressed to the CS are not likely to have been influenced by summation of contextinduced fear with fear of the CS.

The mean suppression ratios for the test CS are illustrated in Figure 1. Greater suppression in response to the CS (conditioned suppression) is denoted by a lower ratio, while no suppression is denoted by a value of .50. This graph suggests that subjects that received CS preexposure suppressed less than control subjects (i.e., the CSpreexposure effect occurred). Figure 1 also suggests that, with an increasing retention interval, suppression decreased in the PE condition but not in the Ctrl condition (i.e., an augmented CS-preexposure effect developed). The following statistical analyses give credence to these conclusions. First, a 2 (treatment: PE vs. Ctrl) $\times$ 2 (retention interval: short vs. long) ANOVA was conducted with the combined suppression ratio from the two test trials as the dependent variable. This analysis revealed main effects of treatment $[F(1,44)=26.85, p<$ $.05]$ and retention interval $[F(1,44)=5.20, p<.05]$. However, the treatment $\times$ retention interval interaction was only marginally significant $[F(1,44)=3.93, p<$ .06].

While the interaction was not significant, there certainly was a tendency $(p<.06)$, suggesting that the retention interval differentially affected the rats in the PE and $\mathrm{Ctrl}$ conditions. Examination of the data from the individual test trials indicated a greater tendency toward an augmented CS-preexposure effect on the first test trial relative to the second. The subjects in the PE-Short group showed suppression $(M=.23 ; S E M=.06)$, while group PE-Long showed no suppression on the first test trial $(M=.50 ; S E M=.03)$, suggesting an increase in the effect of CS preexposure with delayed testing. Both the Ctrl-Short $(M=.15 ; S E M=.06)$ and the Ctrl-Long 


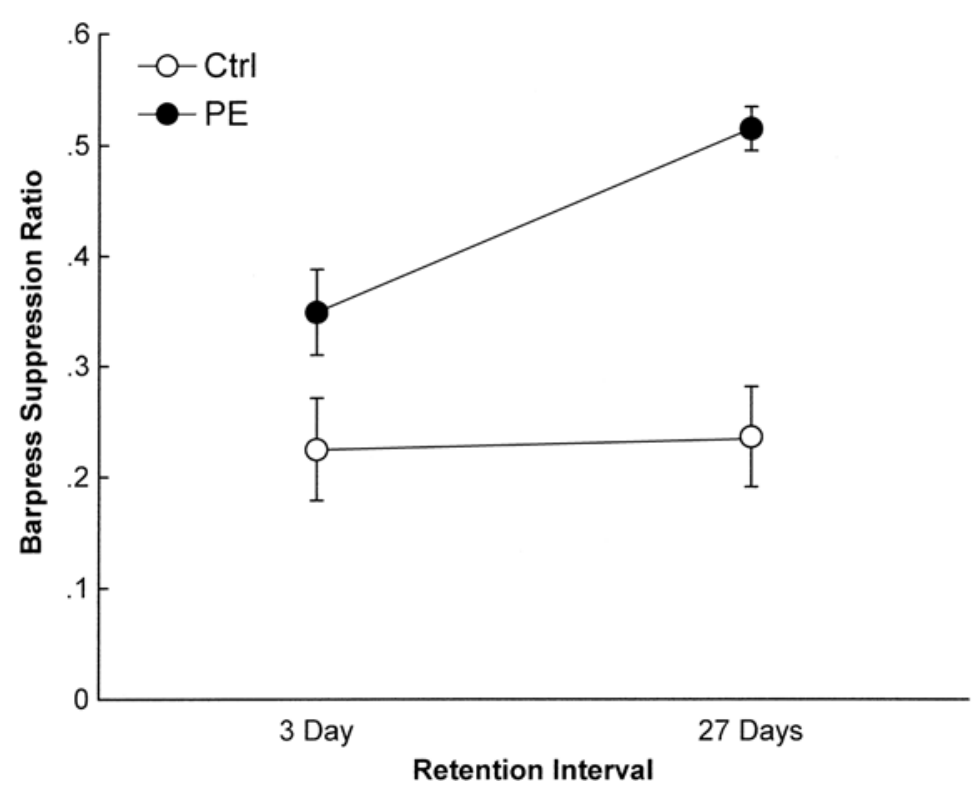

Figure 1. Experiment 1: Augmentation of the CS-preexposure effect. The points depict mean suppression ratios (calculated by pooling barpresses on Test Trials 1 and 2). Lower ratios indicate lower levels of barpressing, thus better conditioned responding to the fear-invoking stimulus. Therefore, higher scores are indicative of the CS-preexposure effect. Error brackets denote the standard error of the mean for each group. See Table 1 for procedural details.

$(M=.12 ; S E M=.05)$ groups suppressed more than the subjects in the PE conditions matched for retention interval. In order to determine whether the augmented CSpreexposure effect was significant on the first test trial, a 2 (treatment: PE vs. Ctrl) $\times 2$ (retention interval: short vs. long) ANOVA was conducted. This analysis yielded a main effect of treatment, retention interval, and an interaction between the two variables $(p s<.05)$. A post hoc Tukey's HSD test was conducted to determine the source of the interaction. The mean suppression observed during the first test trial for Group PE-Long was less than that in Groups PE-Short, Ctrl-Long, and CtrlShort $(p s<.05)$. In addition, Group PE-Short did not differ from Group Ctrl-Short $(p>.50)$. These analyses indicate that, on the basis of the first test trial data alone, a CS-preexposure effect was apparent only after a long retention interval, indicating an augmentation of interference with increasing retention interval and no change in conditioned suppression across the two groups that received no nonreinforced exposure of the CS. Our ability to detect this interaction in the analysis of the two test trials was presumably weakened by data compression of the second test trial that arose from extinction of fear in all groups, caused by the nonreinforced presentation of the target stimulus on the first test trial. Although this extinction effect occluded the augmented CS-preexposure effect in this experiment, it did not pose a similar problem in Experiments 2 and 3. Therefore, only the combined data from the two test trials is analyzed in the subsequent experiments.
Technically, the absence of a CS-preexposure effect on the first test trial after the short retention interval suggests that we may have observed an emergence of a CSpreexposure effect after a long retention interval rather than an augmentation of an existing CS-preexposure effect. It is likely that our use of an equal number of paired and unpaired CS presentations produced a responseretarding effect that was too weak to be detected after a short retention interval without the use of an extinction test. While this is not a traditional demonstration of the CS-preexposure effect, which usually involves retarded conditioned responding during acquisition, it is still clear that CS preexposure led to a retardation of conditioned responding when an extinction test was used. For the sake of simplicity, we continue to refer to the effect as an augmented CS-preexposure effect, while the reader may prefer to view it as the emergence of a CS-preexposure effect.

One interpretation of the observed increase in the CSpreexposure effect with increasing retention interval is that when the subjects were tested in a novel temporal context (i.e., after a long retention interval), retrieval of the memory of the more recent reinforced trials was not facilitated by the temporal context of the $X \rightarrow$ S pairings, and responding tended to reflect the less context specific firstacquired memories of the CS (i.e., X-alone trials). However, the possibility must be considered that the increase in the effect of CS preexposure observed with the long retention interval arose simply from the forgetting of the $\mathrm{S} \rightarrow \mathrm{US}$ association, or from a general weakening of the al- 
ready low suppression in response to the preexposed CS. But, the lack of any parallel decrement in the Ctrl condition (and the extinction condition of Experiment 2) makes this argument implausible.

\section{EXPERIMENT 2 Symmetrical Primacy Effects}

Experiment 1 documented an augmentation of the CSpreexposure effect when a long retention interval was interposed between conditioning and testing. This result extends the generality of the augmented CS-preexposure effect to a Pavlovian conditioning preparation other than CTA. As stated above, one plausible interpretation of the augmented CS-preexposure effect is that the first learned of competing memories becomes more potent with increasing retention intervals (i.e., the primacy principle becomes manifest as recency effects fade with increasing retention intervals). Our preparation provides an opportunity to test this account. Lubow and De la Casa (2002) previously examined this interpretation in their study of the augmented CS-preexposure effect and recovery from extinction. The results of their research suggested that the same parameters that allow for the augmented CSpreexposure effect fail to support spontaneous recovery after extinction. This presents a theoretical problem for the primacy account of the augmented CS-preexposure effect, which states that after recency effects have faded, first-learned memories are more readily expressed than are subsequently acquired contradictory memories (Bouton, 1993). According to the principle of primacy, the nature of the first-learned memory (whether it is that of reinforcement or nonreinforcement) is not important; the same manipulation that causes the augmented CS-preexposure effect should lead to an equal and opposite shift in responding to an extinguished CS.

However, there may be a problem inherent in the CTA preparation that precludes a balanced comparison of the CS-preexposure effect and extinction. In a typical CTA preparation, the subjects control the duration and amount of CS exposure; that is, the amount of exposure is dependent on the subjects' ingestive behavior. In a CSpreexposure procedure, the subjects readily consume the CS during the nonreinforced presentations because they have yet to acquire an aversion to the tastant. In a similar extinction design, subjects acquire an aversion to the CS prior to the nonreinforced exposure, which results in relatively little CS consumption over a small number of extinction trials. This potential difference in nonreinforced CS exposure could result in a difference in the efficacy of the CS-preexposure and extinction treatments. In Lubow and De la Casa's (2002) article, a difference in mean CS consumption during nonreinforced trials was not statistically tested, but a potential difference was apparent in a cross-experiment comparison, with subjects in the preexposed condition consuming $8.05 \mathrm{ml}$ compared with $1.44 \mathrm{ml}$ consumed by subjects in the comparable extinction condition. The authors acknowledged that the weak- ness of the extinction manipulation (possibly caused by insufficient CS exposure during extinction trials) might have been the reason for the absence of spontaneous recovery following extinction treatment.

The conditioned barpress suppression preparation avoids this problem because the amount of CS exposure on any given trial is not dependent on the subject's behavior. Experiment 2 was designed to take advantage of this characteristic of the conditioned barpress suppression preparation and examine the effects of delayed testing on subjects that receive CS preexposure prior to reinforcement and subjects that receive extinction trials following reinforced training. As previously mentioned, the principle of primacy predicts opposite shifts in responding following a long retention interval: increasing effect of CS preexposure but a spontaneous recovery from extinction. Subjects in the preexposed and extinction conditions were given identical numbers of paired and unpaired trials; only the trial order varied between groups. Paired-only control groups were unnecessary because the focus of the experiment was on changes in responding that occur over a long retention interval, and the control groups in Experiment 1 showed no change in responding across the two retention intervals used in this experiment.

Method
Subjects
The subjects were 24 male and 24 female rats of the same stock
as those used in Experiment 1 . The subjects were all housed, handled,
and cared for in the same fashion as in Experiment 1. Body-weight
ranges were $260-347 \mathrm{~g}$ for males and $199-235 \mathrm{~g}$ for females. Subjects
were randomly assigned to one of four groups ( $n=12$ for each group),
counterbalanced within groups for sex. The groups were designated
preexposure-short retention interval (PE-Short), preexposure-long
retention interval (PE-Long), extinction-short retention interval
(Ext-Short), and extinction-long retention interval (Ext-Long).

\section{Apparatus}

The apparatus and the stimulus parameters used in this experiment were identical to those of Experiment 1 . However, because the design of the present experiment required the use of only one CS, the complex tone was not used in this study. CS X was always the click train.

\section{Acclimation and Shaping}

The acclimation and shaping procedure was conducted on the first 4 days of the experiment, as described in Experiment 1.

Table 2

Design Summary of Experiment 2

\begin{tabular}{llllrc}
\hline Group & Phase 1 & Phase 2 & Phase 3 & $\begin{array}{r}\text { Retention } \\
\text { Interval }\end{array}$ & Test \\
\hline PE-Short & $12 \mathrm{X}-$ & $12 \mathrm{X} \rightarrow \mathrm{S}$ & $4 \mathrm{~S} \rightarrow \mathrm{US}$ & 3 days & $\mathrm{X}$ \\
PE-Long & $12 \mathrm{X}-$ & $12 \mathrm{X} \rightarrow \mathrm{S}$ & $4 \mathrm{~S} \rightarrow \mathrm{US}$ & 27 days & $\mathrm{X}$ \\
Ext-Short & $12 \mathrm{X} \rightarrow \mathrm{S}$ & $12 \mathrm{X}-$ & $4 \mathrm{~S} \rightarrow \mathrm{US}$ & 3 days & $\mathrm{X}$ \\
Ext-Long & $12 \mathrm{X} \rightarrow \mathrm{S}$ & $12 \mathrm{X}-$ & $4 \mathrm{~S} \rightarrow \mathrm{US}$ & 27 days & $\mathrm{X}$ \\
\hline
\end{tabular}

Note-PE, preexposure; Ext, extinction; X, 60-sec click; S, 5-sec flashing light; US, footshock. Testing consisted of nonreinforced presentations of the target cue. 


\section{Procedure}

The design of Experiment 2 is displayed in Table 2. Subjects in the PE condition received Phase 1 and 2 training identical to that in Experiment 1. Subjects in the Ext condition received similar training with the order of the phases reversed. Phase 3 and testing occurred as in Experiment 1.

Phase 1. On Days 5 and 6, Phase 1 training occurred in 60-min sessions. For the subjects in the two PE groups, each day included six X-only presentations separated by $9( \pm 4.5)$-min ITIs. For the subjects in the two Ext groups, each day included six $\mathrm{X} \rightarrow \mathrm{S}$ pairings separated by $9( \pm 4.5)$-min ITIs. The onset of the 5 -sec Stimulus $S$ occurred at the end of the 60 -sec CS X.

Phase 2. On Days 7 and 8, Phase 2 also consisted of daily 60-min sessions. For the PE groups, the session included six daily $\mathrm{X} \rightarrow \mathrm{S}$ pairings again separated by $9 \pm 4.5$-min ITIs. The Ext groups received six daily exposures to $\mathrm{X}$ alone.

Phase 3: First-order conditioning of the surrogate stimulus. On Day 9, all subjects received four $\mathrm{S} \rightarrow \mathrm{US}$ pairings in a $60-\mathrm{min}$ session. These trials were separated by $15( \pm 7.5)$-min ITIs. Again, the onset of the US occurred at the termination of surrogate Stimulus $\mathrm{S}$.

Short retention interval reshaping and testing. On Days 10 and 11, the subjects from the PE-Short and Ext-Short groups were given daily 60-min sessions of uninterrupted barpressing, as in Experiment 1 . On Day 12, subjects from the two short retention interval groups were tested on CS X in a 30-min test session. This test session was identical to that used in Experiment 1. The suppression ratio was calculated as in Experiment 1.

Long retention interval reshaping and testing. As in Experiment 1 , all subjects in the long retention interval condition were given 2 days of reshaping on Days 34 and 35. On Day 36, these subjects were tested on responding to $\mathrm{X}$.

\section{Results and Discussion}

During training, 2 subjects (one each from Groups Ext-Long and Ext-Short) became ill and were subse- quently eliminated from the experiment without being tested. A 2 (treatment: Ext vs. PE) $\times 2$ (retention interval: short vs. long) ANOVA was performed using the number of pre-CS barpresses as the dependent variable. No significant differences were detected, indicating no appreciable differences in the subjects' fear of the context $(p s>.05)$. Mean barpress suppression ratios for the $\mathrm{CS}$ are presented in Figure 2. The figure suggests an interaction of trial order (extinction vs. CS preexposure) with retention interval (long vs. short). That is, over the increasing retention interval, the effect of CS preexposure waxed and extinction waned. The following statistics were conducted to assess these impressions.

First, a 2 (treatment: Ext vs. PE) $\times 2$ (retention interval: short vs. long) ANOVA was performed to determine whether there were any group differences in responding to the target CS using the raw data pooled from each group's two test trials. There was an effect of treatment $[F(1,42)=6.69, p<.05]$ and retention interval $[F(1,42)=$ $8.72, p<.05]$, and an interaction $[F(1,42)=40.54, p<$ $.05]$. A planned comparison revealed greater suppression in Group PE-Short than in Group PE-Long, thereby replicating the augmented CS-preexposure effect seen in Experiment $1[F(1,42)=6.09, p<.05]$. This augmented CS-preexposure effect was observed in concert with an increase in suppression in Group Ext-Long relative to Group Ext-Short $[F(1,42)=41.63, p<.05]$; that is, strong spontaneous recovery from extinction was observed. This result lends support to the view that the augmented CS-preexposure effect observed in Experiment 1 was indeed due to a primacy effect and not to alternative factors such as a degradation of the $\mathrm{S} \rightarrow \mathrm{US}$ as-

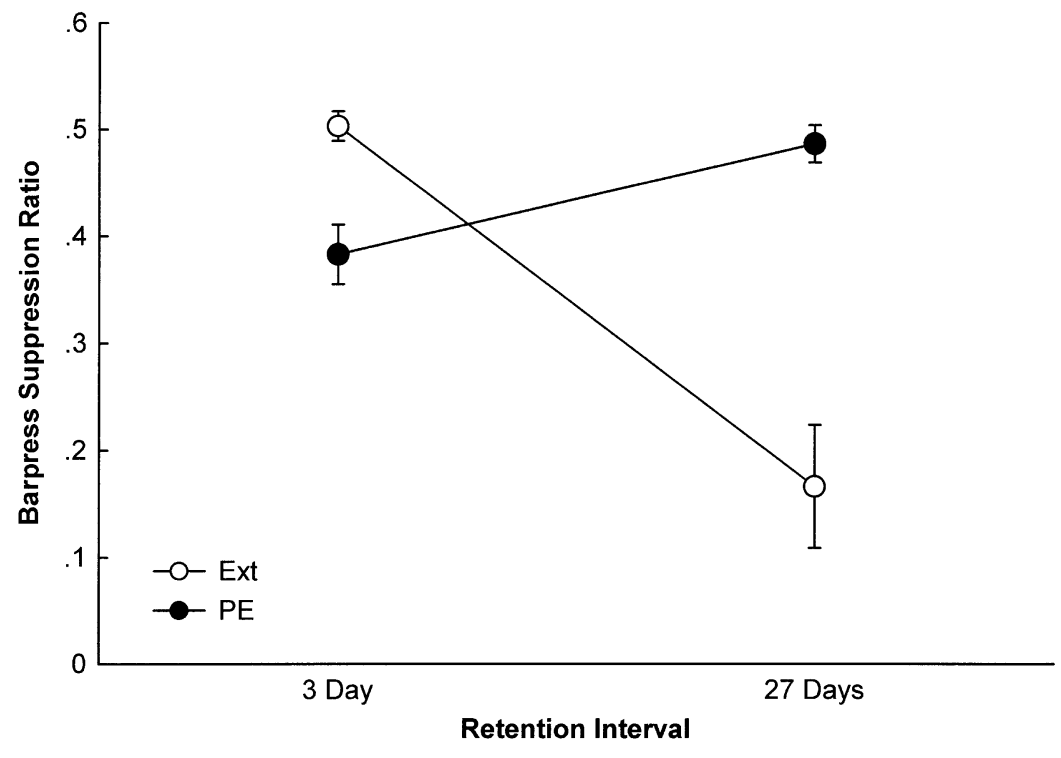

Figure 2. Experiment 2: Symmetrical primacy effects. The points depict mean suppression ratios (calculated by pooling barpresses on Test Trials 1 and 2). Lower ratios indicate less barpressing, thus better conditioned responding to the fear-invoking stimulus. Error brackets denote the standard error of the mean for each group. See Table 2 for procedural details. 
sociation or a spontaneous alleviation of already weak suppression. Suppression in response to the extinguished CS was very weak when tested at a short retention interval, and if either of the alternative factors had been active, it would only have deteriorated further. Instead, a recovery of suppression was observed, which further supports the idea that the first-acquired memories of the CS are more strongly expressed following a long retention interval. While there was no continuously paired control (i.e., the Ctrl condition of Experiment 1; Table 1) for spontaneous recovery in this experiment, there was clearly an increase in suppression in response to the extinguished CS when tested after a long retention interval. Examination of the control group used in Experiment 1 reveals no such increase for a continuously reinforced stimulus (comparing Groups Ctrl-Short and Ctrl-Long).

\section{EXPERIMENT 3 Priming of Symmetrical Primacy Effects}

In Experiment 2, we observed symmetrical shifts toward primacy when a stimulus was tested after a long retention interval following extinction and CS-preexposure training. These data are consistent with interference theories of the CS-preexposure effect and spontaneous recovery if it is assumed that expression of the second learned memory concerning a CS is facilitated by testing in the temporal context in which the memory was acquired (i.e., a short retention interval; e.g., Bouton, 1993). Experiment 3 was designed to test another prediction of Bouton's (1993) interference theory. Specifically, if the present augmented CS-preexposure and spontaneous recovery from extinction effects result from interactions between interfering memories and contextual priming, then both effects should be sensitive to shifts in the physical context as well as the temporal context. According to Bouton's interference theory of the CS-preexposure effect and extinction, the degree to which a particular memory is expressed can be determined by the contextual cues (physical or temporal) present at the time of testing (e.g., Brooks, 2000; Brooks, Palmatier, Garcia, \& Johnson, 1999). The contextual cues present during different phases of training can at test prime a particular memory of the CS, making it more easily retrieved if the CS is presented soon after the prime is presented.

Experiment 3 was conducted to determine whether the effects of recency could be attenuated or augmented with a direct priming manipulation. If the level of responding is truly dependent upon contextual priming, then a direct priming manipulation should create effects similar to those seen when a retention interval is interposed between testing and training. In order to prime the different hypothetical CS-outcome associations, this design used punctate priming stimuli embedded in each of the training phases. Prior to the test of $\mathrm{X}$, the priming stimulus from either Phase 1 (Stimulus A) or Phase 2 (Stimulus B) was presented. If the previously observed effects were due to priming by the temporal context, then simi- lar results would be expected to occur in this design. That is, with a short retention interval, priming the Phase 1 training should produce a spontaneous recovery from extinction and an augmented effect of CS-preexposure treatment, while Phase 2 priming should produce no change, or an even greater recency effect.

\section{Method}

\section{Subjects}

The subjects were 36 male and 36 female rats of the same stock as those used in the previous experiments. Body-weight ranges were $242-316 \mathrm{~g}$ for males and $180-226 \mathrm{~g}$ for females. The subjects were all housed, handled, and cared for in the same fashion as in the prior experiments. Subjects were randomly assigned to one of six groups ( $n=12$ for each group), counterbalanced within groups for sex. The groups were designated preexposure-Prime A (PE-PrimeA), preexposure-no prime (PE-NoPrime), preexposure-Prime B (PEPrimeB), extinction-Prime A (Ext-PrimeA), extinction-no prime (Ext-NoPrime), and extinction-Prime B (Ext-PrimeB).

\section{Apparatus}

The apparatus used in this experiment was identical to that used in Experiments 1 and 2. As in Experiment 2, CS X was always the click train. In this experiment, the priming Stimuli A and B were a buzzer and the complex tone (consisting of $3000-\mathrm{Hz}$ and $3200-\mathrm{Hz}$ pure tones), both presented at $8 \mathrm{~dB}$ above background, counterbalanced within groups.

\section{Acclimation and Shaping}

The acclimation and shaping procedure was conducted as described in the prior experiments, with the addition of an extra day of shaping consisting of a repetition of the Day 1 barpressing training regimen (a fixed-time 2 -min schedule combined with a continuous reinforcement schedule) because several animals did not adequately respond on Day 1.

\section{Procedure}

The design of Experiment 3 is displayed in Table 3. Subjects in the PE and Ext conditions received training similar to that in Experiment 2, except that Phase 1 featured unpaired presentations of Priming Stimulus A, and Phase 2 included presentations of Priming Stimulus B. All testing occurred after a short retention interval. Subjects in the Prime A condition received a presentation of A before test, while those in the Prime $\mathrm{B}$ condition received $\mathrm{B}$, and those in the NoPrime condition received no priming stimulus presentation before test.

Phase 1. Phase 1 training occurred in daily 60 -min sessions on Days 6 and 7. For the subjects in the three PE groups, each day included six X-only presentations intermixed with six A-only pre-

Table 3

Design Summary of Experiment 3

\begin{tabular}{llccr}
\hline \multicolumn{1}{c}{ Group } & \multicolumn{1}{c}{ Phase 1} & \multicolumn{1}{c}{ Phase 2} & Phase 3 & Test \\
\hline PE-PrimeA & $12 \mathrm{X}-/ 12 \mathrm{~A}$ & $12 \mathrm{X} \rightarrow \mathrm{S} / 12 \mathrm{~B}$ & $4 \mathrm{~S} \rightarrow \mathrm{US}$ & $\mathrm{A} \ldots \mathrm{X}$ \\
PE-NoPrime & $12 \mathrm{X}-/ 12 \mathrm{~A}$ & $12 \mathrm{X} \rightarrow \mathrm{S} / 12 \mathrm{~B}$ & $4 \mathrm{~S} \rightarrow \mathrm{US}$ & $\mathrm{X}$ \\
PE-PrimeB & $12 \mathrm{X}-/ 12 \mathrm{~A}$ & $12 \mathrm{X} \rightarrow \mathrm{S} / 12 \mathrm{~B}$ & $4 \mathrm{~S} \rightarrow \mathrm{US}$ & $\mathrm{B} \ldots \mathrm{X}$ \\
Ext-PrimeA & $12 \mathrm{X} \rightarrow \mathrm{S} / 12 \mathrm{~A}$ & $12 \mathrm{X}-/ 12 \mathrm{~B}$ & $4 \mathrm{~S} \rightarrow \mathrm{US}$ & $\mathrm{A} \ldots \mathrm{X}$ \\
Ext-NoPrime & $12 \mathrm{X} \rightarrow \mathrm{S} / 12 \mathrm{~A}$ & $12 \mathrm{X}-/ 12 \mathrm{~B}$ & $4 \mathrm{~S} \rightarrow \mathrm{US}$ & $\mathrm{X}$ \\
Ext-PrimeB & $12 \mathrm{X} \rightarrow \mathrm{S} / 12 \mathrm{~A}$ & $12 \mathrm{X}-/ 12 \mathrm{~B}$ & $4 \mathrm{~S} \rightarrow \mathrm{US}$ & $\mathrm{B} \ldots \mathrm{X}$ \\
\hline
\end{tabular}

Note-PE, preexposure; Ext, extinction; PrimeA, priming Phase 1 at test; PrimeB, priming Phase 2 at test; NoPrime, no priming at test; X, 60-sec clicker; S, 5-sec flashing light; A and B, 30-sec buzzer and highfrequency mixed tone; US, footshock. Testing consisted of two nonreinforced presentations of the target cue. 
sentations (specifically, X, A, X, X, A, X, A, A, X, A, A, X). For the rats in the three Ext groups, each session included six $X \rightarrow S$ pairings intermixed with six A-only presentations (specifically, $X \rightarrow S, A, X \rightarrow S, X \rightarrow S, A, X \rightarrow S, A, A, X \rightarrow S, A, A, X \rightarrow S$ ). In both groups, the presentations of Stimuli $X$ and $A$ were separated by a $4( \pm 1)$-min ITI. Priming Stimulus A was $30 \mathrm{sec}$ in duration. As in the previous two experiments, the onset of the 5-sec Stimulus S occurred at the end of the $60-\sec$ CS X.

Phase 2. Phase 2 consisted of daily 60 -min sessions on Days 8 and 9. For the $P E$ groups, the session included six $X \rightarrow S$ pairings intermixed with six B-only presentations (the order of presentation and the ITIs were identical to those received by the Ext groups in Phase 1). The Ext groups received six X-only presentations intermixed with six B-only presentations (the order and ITIs were identical to those received by the PE groups in Phase 1). Priming Stimulus B was $30 \mathrm{sec}$ in duration.

Phase 3: First-order conditioning of the surrogate stimulus. First-order conditioning of the surrogate stimulus on Day 10 was identical to that conducted in the prior experiments.

Reshaping and testing. On Days 11 and 12, all subjects were given daily 60 -min sessions of uninterrupted barpressing. Subjects that made fewer than 50 responses in a session were each given an extra 30-min session in a day. On Day 13, all groups were tested on CS X in a $30-m i n$ test session. Three minutes into each session, there was a $30-\mathrm{sec}$ presentation of Priming Stimulus A (PrimeA condition), a $30-\mathrm{sec}$ presentation of Priming Stimulus B (PrimeB condition), or nothing (NoPrime condition). Seven minutes into the session (4 min after the onset of the priming stimuli), there was a nonreinforced presentation of CS X (60 sec in duration). Eleven minutes into the session, there was another presentation of the priming stimulus for the appropriate groups. This was followed by a second nonreinforced presentation of X 15 min into the session. The suppression ratio was calculated as in Experiment 1.

\section{Results and Discussion}

A 2 (treatment: PE vs. Ext) $\times 3$ (prime: PrimeA vs. PrimeB vs. NoPrime) ANOVA conducted on the pre-CS scores did not reveal any significant differences in baseline rates of leverpressing ( $p \mathrm{~s}>.50)$. Mean suppression ratios are depicted in Figure 3. Most apparent is an interaction between the priming stimulus at test (A vs. B) and treatment (Ext vs. PE). Seemingly, priming the Phase 1 memory with Stimulus A induced more suppression to $\mathrm{X}$ in the extinction condition than in the CS-preexposure condition, whereas priming the Phase 2 memory with Stimulus B induced more suppression to $\mathrm{X}$ in the preexposed condition than in the extinction condition. Thus, Priming Stimulus B produced a recency effect and Priming Stimulus A produced a primacy effect, despite equal retention intervals in this experiment. The following statistical analysis corroborates these conclusions.

A 2 (treatment) $\times 3$ (prime) ANOVA conducted on the combined suppression ratio from the two tests of CS X revealed main effects of treatment $[F(1,66)=29.50, p<$

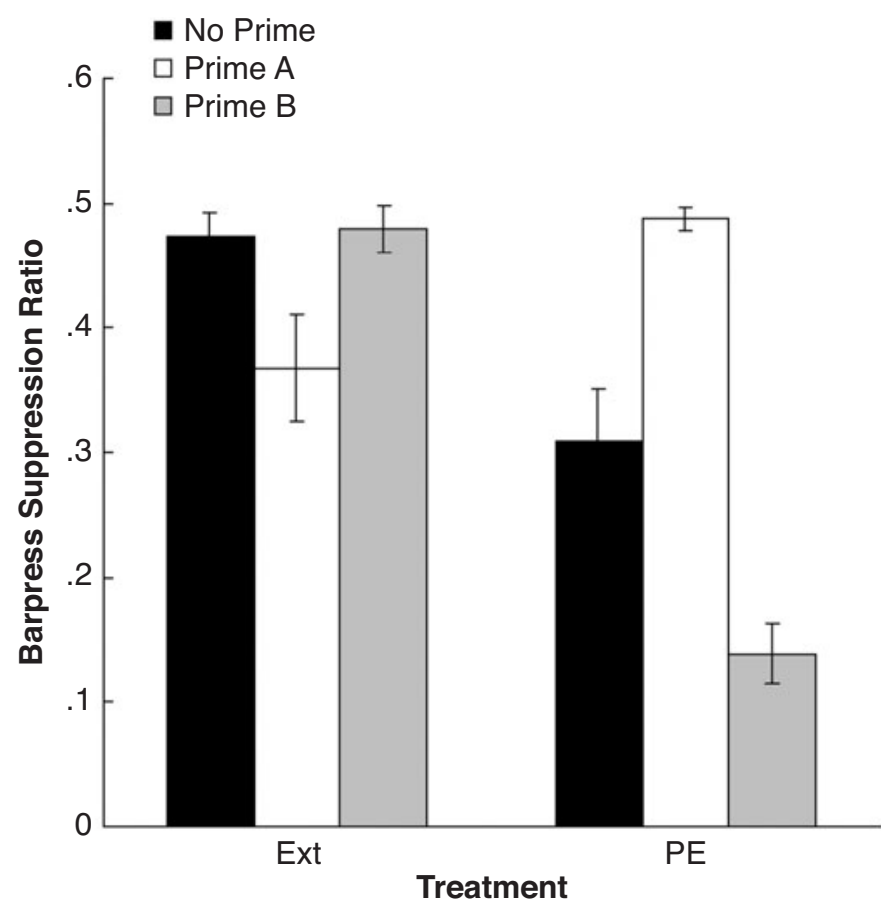

Figure 3. Experiment 3: Priming of symmetrical primacy effects. Ext groups received $X$-alone trials after $X \rightarrow S$ pairings. PE groups received $\mathrm{X}$-alone trials before $\mathrm{X} \rightarrow \mathrm{S}$ pairings. The bars depict mean suppression ratios (calculated by pooling barpresses on Test Trials 1 and 2). Lower scores indicate less barpressing, thus better conditioned responding to the fear-invoking stimulus. Error brackets denote the standard error of the mean for each group. See Table 3 for procedural details. 
$.05]$, prime $[F(2,66)=31.84, p<.05]$, and an interaction between the two variables $[F(2,66)=8.87, p<$ $.05]$. Planned comparisons were conducted to determine the specific group differences responsible for the interaction. Subjects trained with the PE treatment suppressed less to the CS when the Phase 1 training was primed (PEPrimeA) than subjects that received no priming stimulus prior to test (PE-NoPrime), indicating an augmented effect of CS preexposure caused by punctate stimulus priming $[F(1,66)=18.96, p<.05]$. Conversely, a recovery from the CS-preexposure effect was observed when Phase 2 training was primed prior to test $[F(1,66)=17.07$, $p<.05$ ] (PE-PrimeB vs. PE-NoPrime). In the Ext condition, a recovery of suppression was seen when the first phase of training was primed before testing $[F(1,66)=$ $6.64, p<.05]$ (Ext-PrimeA vs. Ext-NoPrime). However, a symmetrical augmented extinction effect was not apparent, likely due to a floor effect in responding $[F(1,66)=$ $0.02, p>.80$ ] (Ext-PrimeB vs. Ext-NoPrime). That is, Group Ext-NoPrime had a suppression ratio so close to .50 that there was no room to see any possible reduction in suppression.

\section{GENERAL DISCUSSION}

These findings demonstrate that it is possible to simultaneously observe primacy effects in both the effects of CS-preexposure and extinction treatments when the retention interval occurs outside of the training context. De la Casa and Lubow $(2000,2002)$ have repeatedly demonstrated the augmentation of the CS-preexposure effect using a CTA preparation, whereas the present series of experiments demonstrates the effect using a conditioned barpress suppression preparation. The results of Experiment 2 suggest that this effect is due to a shift from recency to primacy that occurs during a long retention interval as opposed to a general decline in responding that is already weak. That is, when tested immediately after training, subjects behaved in a way that was congruent with the most recent trial types $(\mathrm{X} \rightarrow \mathrm{S}$ for the PE-Short group, and X-alone trials for the Ext-Short group). Conversely, subjects tested after a long retention interval responded in a way that was more congruent with their first experiences with the CS than their most recent experiences. The results of Experiment 3 indicate that the effect of interposing a retention interval immediately before testing can be simulated by using priming cues to activate the different memories concerning CS $\mathrm{X}$, instead of a shift in the temporal context. Furthermore, memories of both the paired and X-alone representations of the target stimulus were subject to facilitated retrieval by the punctate priming stimuli, further emphasizing the flexibility of responding depending upon priming of different memories of the CS. These results have some intriguing theoretical implications.

To this point, the present series of experiments has been discussed in terms of interference theories of the
CS-preexposure effect and extinction (e.g., Bouton, 1993; Miller et al., 1986; Spear, 1978). Certain attention-focused theories of the CS-preexposure effect and extinction also predict the augmented CS-preexposure effect and spontaneous recovery. For example, Lubow's (1989) conditioned attention theory (CAT) includes a mechanism that allows for the spontaneous recovery of conditioned inattention. According to CAT, CS preexposure produces a conditioned attention deficit to the CS. The theory assumes that an animal will devote a baseline level of attention to any novel stimulus, and this baseline level may be elevated (through CS-US pairings) or lowered (by CS-alone exposures). Essentially, CS-alone exposures serve as CS-no US pairings, which result in a conditioned decrease in the normal level of attention that an animal would direct toward a novel stimulus. Because the attention deficit is conditioned, the theory indicates that it is subject to the same influences as a classically conditioned stimulus (i.e., it may be extinguished, blocked, overshadowed, etc.). The attention deficit acquired during preexposure retards subsequent learning of the CS-US association, resulting in slower acquisition. However, the attention deficit is steadily extinguished during reinforcement, as subjects learn to attend to the now reinforced CS. Lubow has suggested that when an attention deficit is acquired and then extinguished, it may be recovered after a long retention interval through the same mechanism that produces a spontaneous recovery of conditioned responding after extinction. Therefore, CAT predicts the augmentation of the CS-preexposure effect observed in Experiment 1 on the basis of spontaneous recovery of conditioned inattention.

Spontaneous recovery from extinction can be explained by an unconditioned attention-focused theory. For example, Robbins (1990; see also Pavlov, 1927) has suggested that extinction results from decreased attention to a CS. In turn, spontaneous recovery of responding is caused by a natural renewal of attention over time. This account of spontaneous recovery from extinction is sufficient to explain the spontaneous recovery effect observed in Experiment 2, but it is clearly incompatible with the CAT explanation of the augmented of CS-preexposure effect. Specifically, Robbins has suggested that attention to a CS increases as time passes, while Lubow (1989) has suggested the opposite. Also, Lubow has indicated that conditioned inattention (as opposed to attention) can be spontaneously recovered through an undefined mechanism that would certainly conflict with Robbins's account of spontaneous recovery from extinction. Thus, while both theories suggest that the CS-preexposure effect and extinction result from an attention deficit, they disagree about the way that attention fluctuates as time passes. The theories could be integrated if a mechanism for primacy were included, but such a mechanism may be difficult to incorporate into these theories.

Instead of resulting from an attention deficit, the present effects of extinction and CS-preexposure treatment 
are probably best viewed as a result of interference between different memories concerning the CS. This idea is well explained in Bouton's (1993) review, in which the effects of both CS preexposure and extinction are explained via competition between different memories concerning the target stimulus, one reinforced and one not reinforced. Bouton postulated that the expression of a given CS memory is modulated by the context of test, and that the contradictory memories of the CS are differentially subject to occasion setting by the context. Also, both time and physical stimuli present during training and the time of training constitute the context. At a test of a stimulus, the CS-alone memory interferes with the expression of the CS-outcome memory, resulting in retarded conditioned responding. This response-reducing interference may be alleviated through contextual manipulations (e.g., McLaren, Bennett, Plaisted, Aitken, \& Mackintosh, 1994), punctate priming manipulations (e.g., Experiment 3), and temporal manipulations (e.g., Experiment 2; Bakner, Strohen, Nordeen, \& Riccio, 1991). The interference can also be augmented through punctate priming of the nonreinforced trials (e.g., Experiment 3 ) and temporal manipulations (e.g., De la Casa \& Lubow, 2000). While all of these results support the view that the CS-preexposure effect is due to a transient retrieval deficit rather than a permanent deficit in acquisition, the conflicting results involving retention interval effects require additional explanation concerning the details of contextual modulation.

The attenuation of the CS-preexposure effect following a retention interval is best explained by the theories that predict memories of reinforcement to be more temporally stable and less context specific than memories of nonreinforcement (e.g., Bouton, 1993). Further research conducted by Bouton and Nelson (1994), however, has indicated that this theory cannot accurately describe all of the pertinent data (including the augmented CSpreexposure effect). More appropriate for the present data (also proposed by Bouton, 1993; and formally stated more recently by Nelson, 2002) is the proposal that ambiguous CS memories are more context specific than unambiguous CS memories. This view is predicated on the assumption that the first-learned memories of a consistently reinforced or nonreinforced CS present an animal with an unambiguous response decision. However, once the animal is exposed to contradictory training with the same CS (by withdrawing, adding, or changing the outcome, as was done in Phase 2 in Experiments 1-3), the $\mathrm{CS}$ becomes ambiguous because there is no consistently appropriate response. Therefore, the animal encodes the contradictory training as specific to the context of training in order to disambiguate the CS. In this way, the second association is only strongly manifest when it is primed by the context in which it was learned.

Bouton's theory concerning primacy is consistent with both the findings of De la Casa and Lubow and the present experiments, in which the temporal context is ma- nipulated prior to test. The theory suggests that the passage of time acts as a context shift (possibly by causing changes in the animal's internal state), resulting in an enhanced expression of the less context dependent firstlearned memories relative to the more context dependent second-learned memories. In further support of this theory, similar results have been observed in experiments employing a physical context shift analogous to the imposition of a retention interval before testing. When subjects receive CS preexposure and training in one physical context, and testing in a novel context, responding is weaker than similarly preexposed and trained subjects that are tested in the training context (Swartzentruber \& Bouton, 1992; Westbrook, Jones, Bailey, \& Harris, 2000). Therefore, shifting either the physical or temporal context prior to test produces a similar augmented effect of CS preexposure, which supports both the view that long retention intervals cause a shift in the temporal context and the theory that responding to an ambiguous stimulus will reflect the first-acquired memories concerning that stimulus when the stimulus is presented in a neutral context.

Although Experiments 1 and 2 show that the firstlearned memories of a CS are less context dependent relative to second-learned memories concerning the same CS, it is also important to note that even first-learned memories were subject to contextual modulation in Experiment 3. In that experiment, the CS-preexposure effect was augmented by the presentation of the Phase 1 priming stimulus before test and attenuated by the presentation of the Phase 2 priming stimulus. Such results indicate that the expression of both first- and second-learned memories is subject to contextual modulation. The ubiquity of contextual modulation is important to consider when one is interpreting results from other experiments (e.g., Aguado et al., 1994) that show a recovery from the CS-preexposure effect when a delay is imposed between CS preexposure and training (as distinct from that between training and testing, which was the case in Experiments 1 and 2). If the first-learned memories of a CS were entirely context independent, then a shift in temporal context between CS preexposure and CS reinforcement should have no effect on the detection of the CS-preexposure effect. Experiment 3 shows that first-learned memories of the CS are subject to some contextual modulation and could only be less context specific relative to the second-learned memories concerning the CS.

Whereas the simultaneous observation of spontaneous recovery and an augmented effect of CS-preexposure treatment certainly supports the principle of primacy (Konorski \& Szwejkowska, 1952), it is important to recognize that there is evidence of another, possibly conflicting, mechanism. Evidence of a recovery from the CS-preexposure effect obtained in a first-order conditioned fear preparation supports the view that memories of reinforcement are stronger and more durable than memories of nonreinforcement (e.g., Killcross et al., 1998; Kraemer et al., 1991). Perhaps central to the dif- 
ference in the effect of retention interval between the present research and those conditioned suppression studies that precluded extinction of the training context is our use of a preparation in which the unpaired and paired trials did not differ in biological significance (in contrast to first-order fear conditioning).

The immediate objection to this assertion comes from De la Casa and Lubow's research, in which the augmented CS-preexposure effect was observed in a first-order conditioning preparation. However, while their studies were certainly conducted with a biologically significant outcome (i.e., an illness-inducing injection), the nonreinforced CS exposures may have also involved a biologically significant outcome. As previously mentioned, the CTA preparation often uses water-deprived rats as subjects and tastants dissolved in solution as CSs. Because nonreinforced exposures to a given stimulus still involve the taste being paired with positive postingestive feedback (e.g., satiation of thirst), these CS exposures are presumably highly salient and important to the subjects. Essentially, fluid CSs themselves may be treated as potent USs (for a review of this position, see Garcia, 1989). In this framework, the CS-preexposure effect and extinction in a conditioned taste aversion preparation may be viewed as examples of counterconditioning. That is, the CS is paired with an appetitive outcome on so-called nonreinforced trials, and paired with an aversive outcome on so-called reinforced trials. Interestingly, a long retention interval imposed before testing can produce a shift toward primacy in responding to appetitive-aversive counterconditioned stimuli (Bouton \& Peck, 1992). Thus, during the CS-outcome pairings in both the present retention interval studies and those of De la Casa and Lubow, the CS may have been of little more significance than on the nonreinforced trials. Without a difference in significance between the CS on the reinforced trials and nonreinforced trials, this experimental strategy should favor the operation of the primacy principle, simply because there is no difference in importance that could be used to guide responding. In short, the primary mechanism that determines responding to an ambiguous stimulus in a novel context may be dependent on nature of the training. However, the effect of relative CS importance was not explicitly tested in this series. Further experiments are being conducted in our laboratory to address this issue.

\section{REFERENCES}

Aguado, L., Symonds, M., \& Hall, G. (1994). Interval between preexposure and test determines the magnitude of latent inhibition: Implications for an interference account. Animal Learning \& Behavior, 22, 188-194.

Bakner, L., Strohen, K., Nordeen, M., \& Riccio, D. C. (1991). Postconditioning recovery from the latent inhibition effect in conditioned taste aversion. Physiology \& Behavior, 50, 1269-1272.

Bouton, M. E. (1993). Context, time, and memory retrieval in the interference paradigms of Pavlovian learning. Psychological Bulletin, 114, 80-99.

BOUTON, M. E., \& NeLSON, J. B. (1994). Contextual specificity of target versus feature inhibition in feature negative discrimination. Journal of Experimental Psychology: Animal Behavior Processes, 20, 51-65. Bouton, M. E., \& PeCK, C. A. (1992). Spontaneous recovery in crossmotivational transfer (counterconditioning). Animal Learning \& Behavior, 20, 313-321.

BrooKs, D. C. (2000). Recent and remote extinction cues reduce spontaneous recovery. Quarterly Journal of Experimental Psychology: Comparative \& Physiological Psychology, 53B, 25-58.

Brooks, D. C., Palmatier, M. I., Garcia, E. O., \& Johnson, J. L. (1999). An extinction cue reduces spontaneous recovery of a conditioned taste aversion. Animal Learning \& Behavior, 27, 77-88.

De la CASA, L. G., \& LuBow, R. E. (2000). Superlatent inhibition with delayed conditioned taste aversion testing. Animal Learning \& Behavior, 28, 389-399.

De la Casa, L. G., \& Lubow, R. E. (2002). An empirical analysis of the superlatent inhibition effect. Animal Learning \& Behavior, 30, 112120.

Dickinson, A., \& BURKE, J. (1996). Within-compound associations mediate the retrospective revaluation of causality judgements. Quarterly Journal of Experimental Psychology: Comparative \& Physiological Psychology, 49B, 60-80.

Escobar, M., Arcediano, F., \& Miller, R. R. (2002). Latent inhibition and contextual associations. Journal of Experimental Psychology: Animal Behavior Processes, 28, 123-136.

GARCIA, J. (1989). Food for Tolman: Cognition and cathexis in concert. In T. Archer \& L. Nilsson (Eds.), Aversion, avoidance, and anxiety (pp. 45-84). Hillsdale, NJ: Erlbaum.

Grahame, N. J., Barnet, R. C., Gunther, L. M., \& Miller, R. R. (1994). Latent inhibition as a performance deficit resulting from CS-context associations. Animal Learning \& Behavior, 22, 395-408.

Killcross, A. S., Kiernan, M. J., Dwyer, D., \& Westbrook, R. F. (1998). Effects of retention interval on latent inhibition and perceptual learning. Quarterly Journal of Experimental Psychology, 51B, $\underline{59-74 .}$

KONORSKI, J., \& SzWEJKowsKa, G. (1952). Chronic extinction and restoration of conditioned reflexes: IV. The dependence of the course of extinction and restoration of conditioned reflexes on the "history" of the conditioned stimulus. (The principle of the primacy of first training). Acta Biologiae Experimentalis, 16, 95-113.

Kraemer, P. J., \& OsSEnKopp, K.-P. (1986). The effects of flavor preexposure and taste interval on conditioned taste aversion in rats. Bulletin of the Psychonomic Society, 24, 219-222.

Kraemer, P. J., RANDALl, C. K., \& CARbaRY, T. J. (1991). Release from latent inhibition with delayed testing. Animal Learning \& Behavior, 19, 139-145.

Kraemer, P. J., \& Roberts, W. A. (1984). The influence of flavor preexposure and test interval on conditioned taste aversions in the rat. Learning \& Motivation, 15, 259-278.

LuBow, R. E. (1989). Latent inhibition and conditioned attention theory. New York: Cambridge University Press.

Lubow, R. E., \& De La CaSa, L. G. (2002). Superlatent inhibition and spontaneous recovery: Differential effects of pre- and postconditioning CS-alone presentations after long delays in different contexts. $\mathrm{An}$ imal Learning \& Behavior, 30, 376-386.

LuBOw, R. E., Schnur, P., \& RIFKIN, B. (1976). Latent inhibition and conditioned attention theory. Journal of Experimental Psychology: Animal Behavior Processes, 2, 163-174.

Mackintosh, N. J. (1975). A theory of attention: Variations in the associability of stimuli with reinforcement. Psychological Review, $\mathbf{8 2}_{2}$ 276-298.

McLaren, I. P. L., Bennett, C., Plaisted, K., Aitken, M., \& MackINTOSH, N. J. (1994). Latent inhibition, context specificity, and context familiarity. Quarterly Journal of Experimental Psychology: Comparative \& Physiological Psychology, 47B, 387-400.

MiLleR, R. R., KASPROW, W. J., \& SCHACHTMAN, T. R. (1986). Retrieval variability: Sources and consequences. American Journal of Psychology, 99, 145-218.

MiLLER, R. R., \& MATZEL, L. (1988). The comparator hypothesis: A response rule for the expression of associations. In G. H. Bower (Ed.), 
The psychology of learning and motivation: Advances in research and theory (Vol. 22, pp. 51-92). San Diego: Academic Press.

NELSON, J. B. (2002). Context specificity of excitation and inhibition in ambiguous stimuli. Learning \& Motivation, 33, 284-310.

Pavlov, I. P. (1927). Conditioned reflexes. New York: Dover.

Pearce, J. M., \& Hall, G. (1980). A model of Pavlovian learning: Variations in the effectiveness of conditioned but not of unconditioned stimuli. Psychological Review, 87, 532-552.

RoBbins, S. J. (1990). Mechanisms underlying spontaneous recovery in autoshaping. Journal of Experimental Psychology: Animal Behavior Processes, 16, 235-249.

SPEAR, N. E. (1978). The processing of memories: Forgetting and retention. Hillsdale, NJ: Erlbaum.
Stout, S. C., Amundson, J. C., \& Miller, R. R. (2004). Trial order and retention interval in human contingency judgments. Manuscript submitted for publication

SwartZentruber, D., \& Bouton, M. E. (1992). Context sensitivity of conditioned suppression following preexposure to the conditioned stimulus. Animal Learning \& Behavior, 20, 97-103.

Westbrook, R. F., Jones, M. L., BAILEy, G. K., \& HARris, J. A. (2000). Contextual control over conditioned responding in a latent inhibition paradigm. Journal of Experimental Psychology: Animal Behavior Processes, 26, 157-173.

(Manuscript received June 25, 2003;

revision accepted for publication March 15,2004 .) 